\title{
A decision support and planning mobility method for large scale traffic networks*
}

\author{
Stephane Mollier ${ }^{1}$, Maria Laura Delle Monache ${ }^{2}$ and Carlos Canudas-de-Wit ${ }^{3}$
}

\begin{abstract}
This paper presents a new planning and decisionmaking method in large scale traffic networks for predicting how traffic evolves in special events, emergencies and changes in the city mobility demands. The proposed method is based on a 2-D aggregated traffic model for large scale traffic networks [1], [2] which describes traffic evolution as a fluid in two space dimensions. We propose an extension of the model by including additional state density variables, each one associated to a particular layer describing vehicles evolving in different directions. The model is a 2D-PDE described by a system of conservation laws. For this specific case, the resulting PDE is not anymore hyperbolic as typically the LWR model but results in a hybrid hyperbolic-elliptic PDE depending on the density level. In this case, usual numerical schemes may be not valid and often lead to oscillation in the solution. Thus, we consider a high order numerical scheme to improve the numerical solution. Finally, the model is used to predict how the typical traffic evolution will be impacted in particular scenarios like special events or changes in demands. Comparative simulations are provided.
\end{abstract}

\section{INTRODUCTION}

Traffic flow congestion is a ubiquitous issue in many cities in the world. The modeling of traffic allows to make predictions and apply control to improve the state of the traffic network. The estimation and the control of traffic congestion is an important field of research that aims, for instance, to optimize the traveling time of drivers. Two wellknown models are the Dynamic User Equilibrium [3] and the Dynamic Traffic Assignment [4]. With the rise of the learning approaches, data-based models start to be considered, in [5] for example estimation and prediction of traffic are done using Deep-Learning.

To improve traffic congestion, there is a need for new models able to describe precisely more complex situations. Macroscopic models aim to describe traffic with aggregated quantities as the vehicle density, speed and flow. The most common macroscopic model is the LWR model [6], [7], which requires knowledge of a function that links the flow and density called Fundamental Diagram (FD) [8]. This model enables to describe the propagation of traffic on a road. It can be extended to the case of a network by defining models of junctions [9]. However, these models require a lot

\footnotetext{
*This project has received funding from the European Research Council (ERC) under the European Union's Horizon 2020 research and innovation programme (grant agreement 694209).

${ }^{1}$ Univ. Grenoble Alpes, CNRS, Inria, Grenoble INP, GIPSA-LAB 38000 Grenoble, France stephane.mollierlgipsa-lab.fr

${ }^{2}$ Univ. Grenoble Alpes, Inria, CNRS, Grenoble INP, GIPSA-LAB 38000 Grenoble, France ml.dellemonachedinria.fr

${ }^{3}$ Univ. Grenoble Alpes, CNRS, Inria, Grenoble INP, GIPSA-LAB 38000 Grenoble, France carlos.canudas-de-witegipsa-lab.fr
}

of parameters to define each junction. Later on, the traffic research community focuses on aggregated models based on the idea that a FD can be defined at the network level and denote this relation Macroscopic Fundamental Diagram (MFD) [10]. This lead to the creation of reservoir models that describe the accumulation in different parts of a network [11].

In this paper, we do another type of aggregation that aims to approximate traffic flow as fluid in the 2D plane. Mathematically, these models are identical to those of pedestrian [12] but have also some fundamental difference starting from the fact that pedestrians generally evolve in the 2D-plane, whereas the propagation of traffic depends on the underlying network. Some two-dimensional traffic flow models are inspired from pedestrian modeling [13], [14]. Indeed, they keep some assumption of these pedestrian models as the fact that there is no maximum density. More generally, different features have been investigated for 2D models. In [15], authors do not consider the FD but assume that the vehicle velocity is given. In [16], a bi-dimensional model for the urban network that takes into account major arterial independently with 1D model, is described in a discrete setting. The idea of two-dimensional models for traffic is also investigated to represent multi-lane traffic [17]. Recently, 2D models relying on the network, with space-dependent parameters have been developed [1], [2]. A method of validation using microsimulation is presented in [18]. However, all these models allow only the representation of a unique direction of flow. These models have a unique flow direction and to the best of our knowledge the only extension to multiple direction is [19].

One way to accomodate the multiple directions of flow is to extend the existing model in [18] to a system of conservation laws. However, these systems of equations are not always hyperbolic as for the case of two populations of pedestrian evolving in opposite direction [12]. This loss of hyperbolicity can produce instability and oscillation on usual numerical methods. To face these issues, researchers [20] suggest to consider a high order numerical method. One of them, the Weighted Essentially Non-Oscillatory (WENO) [21], are commonly used to solve complex problems with sharp discontinuities.

The contributions of this paper are: to present a numerical method (WENO) to compute the solution of simulation of this 2D with multiple directions of flow traffic models and to show practical applications in different scenarios. The paper is organized as follows. First, the models, the network and the parameters are introduced. Then, the numerical method is 
described. Finally, the results of simulation in several studycases are presented.

\section{DESCRIPTION OF THE TWO DIMENSIONAL MULTILAYER MODEL}

\section{A. The two dimensional and multilayer model}

The model described in this article has two main objectives. The first one is to be able to model traffic on a large urban network. The model is constructed in order to describe the density of vehicles as a two-dimensional fluid, evolving in a domain $\Omega \subset \mathbb{R}^{2}$. The second one is to be able to describe multiple flows of vehicles evolving in different directions. Several layers $\ell \in[1, \ldots, L]$ of density $\rho^{\ell}$ are considered such that each layer represents a different direction of flow. The global density $\rho$ is composed by the density of all the layer $\rho(t, x, y)=\left(\rho^{1}(t, x, y), \ldots, \rho^{L}(t, x, y)\right)$. In practice, we study a simple scenario where two layers are enough to represent the direction of traffic. The model is based on a system of conservation laws and can be described as follows, $\forall t \in \mathbb{R}^{+}, \forall(x, y) \in \Omega$ :

$$
\left\{\begin{array}{l}
\frac{\partial \rho^{\ell_{1}}(t, x, y)}{\partial t}+\nabla \cdot \overrightarrow{\Phi^{\ell_{1}}}(\rho(t, x, y))=0 \\
\frac{\partial \rho^{\ell_{2}}(t, x, y)}{\partial t}+\nabla \cdot \vec{\Phi}^{\ell_{2}}(\rho(t, x, y))=0 \\
\rho^{\ell_{1}}(0, x, y)=\rho_{0}^{\ell_{1}}(x, y) \\
\rho^{\ell_{2}}(0, x, y)=\rho_{0}^{\ell_{2}}(x, y)
\end{array}\right.
$$

where

- $\rho^{\ell_{1}}, \rho^{\ell_{2}}$ are the two dimensional densities of the vehicles for the different layers

- $\rho_{0}^{\ell_{1}}, \rho_{0}^{\ell_{2}}$ are the initial densities of the layers

- $\vec{\Phi}^{\ell_{1}}, \vec{\Phi}^{\ell_{2}}$ are the flows of vehicles with respect to each layer which can develop as follow:

$$
\overrightarrow{\Phi^{\ell}}(\rho(t, x, y))=\rho^{\ell}(t, x, y) \cdot v^{\ell}\left(\Psi^{\ell}(\rho(t, x, y))\right) \cdot \vec{d}_{\theta}^{\ell}(x, y)
$$

with

- $v^{\ell}:\left[0, \rho_{\max }\right] \rightarrow\left[0, v_{\max }\right]$ the velocity magnitude of vehicles of the layer $\ell$ corresponding to the Fundamental Diagram (FD).

- $\vec{d}_{\theta}^{\ell}$ the unit vector direction field of the layer $\ell$ that represents the direction of propagation of the vehicles of this layer.

- The affecting density $\Psi^{\ell}$ that give the level of density among all layer that actually affect the speed of the layer $\ell$.

In practice, we consider the simplest case where the effective density is equal to the sum of the density of the different layers:

$$
\begin{aligned}
& \Psi^{\ell_{1}}, \Psi^{\ell_{2}}:\left[0, \rho_{\max }\right]^{2} \rightarrow\left[0, \rho_{\max }\right] \\
& \Psi^{\ell_{1}}(\rho)=\Psi^{\ell_{2}}(\rho)=\rho^{\ell_{1}}+\rho^{\ell_{2}}
\end{aligned}
$$

Overall, the model have three parameters: the direction field of the two layers $\vec{d}_{\theta}^{\ell_{1}}$ and $\vec{d}_{\theta}^{\ell_{2}}$, the maximum density $\rho_{\max }$ and the maximum velocity $v_{\max }$ that rely all on the considered network.

\section{B. Network considered and parameters of the model}

The network is an artificial network of $25 \times 25$ roads on a grid of $5 \mathrm{~km}^{2}$. The roads are spaced on average by approximately $200 \mathrm{~m}$. This network is close to a Manhattan grid but the nodes (junctions) have been moved with a Gaussian noise to reduce the regularity of the network. The network can be seen in Figure 1.

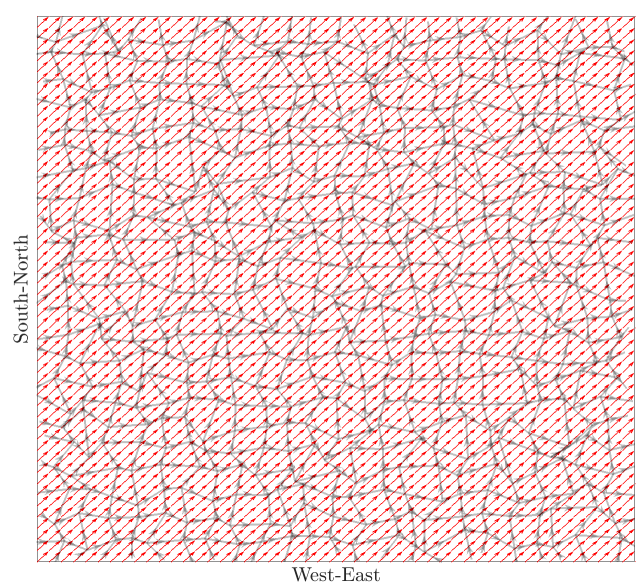

Fig. 1. Field direction of the flux for layer 1 and the considered network

This network contains 1104 links and at this level it starts to be computationally costly to describe it with network models with modeling of junction or with a microscopic approach.

As it has been shown in [2], it is possible to consider space dependent parameters. But, for simplicity, the parameters of the FD have been chosen constant. Also for the same reason, the function chosen for the FD is the one defined by Greenshield as:

$$
v(\rho)=\left(1-\frac{\rho}{\rho_{\max }}\right) v_{\max }
$$

which have the shape represented in Figure 2.

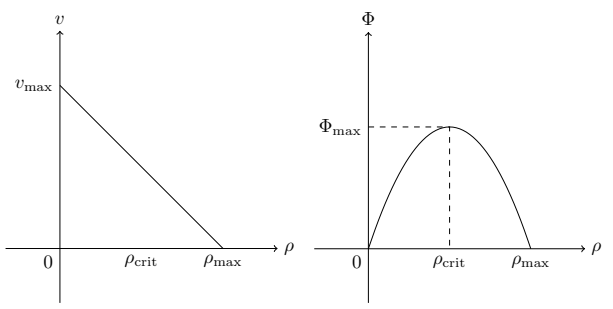

Fig. 2. Fundamental diagram density vs velocity and flux

The direction field of the different layers is defined using the network geometry with the method fully described in [1]. In practice, we consider that there are two main streams of flows. The layer 1 is globally oriented toward the NorthEast direction and is displayed in Figure 1 and the layer 2 is globally oriented toward the North-West direction. 


\section{NUMERICAL METHOD}

\section{A. Properties: two dimensional mixed problem}

Macroscopic continuous traffic models are in general hyperbolic. This is an interesting property because it ensures the finite propagation of information. Thus, it is interesting to study the hyperbolicity of the system (1).

Definition: We denote by $\Phi_{x}$ and $\Phi_{y}$ the projection of the flux function on the $x$ and $y$ dimension axis. Then, the system (1) is hyperbolic if $\forall \alpha \in[0, \ldots, 2 \pi$ [, the matrix:

$$
A=\cos (\alpha) \mathrm{J}_{\Phi_{x}}+\sin (\alpha) \mathrm{J}_{\Phi_{y}}
$$

has real eigenvalues, where $\mathrm{J}_{\Phi_{x}}$ and $\mathrm{J}_{\Phi_{y}}$ are the Jacobian of the flux along $x$ and $y$ dimension.

Thus, if we denote by $\theta^{\ell_{1}}$ and $\theta^{\ell_{2}}$ the angle between the vectors of flow directions, then it is equivalent to show that the determinant $\Delta$ :

$$
\begin{gathered}
\Delta=\left(\cos \left(\alpha-\theta^{\ell_{1}}\right)\left(v^{\ell_{1}}\left(\Psi^{\ell_{1}}(\rho)\right)+\rho^{\ell_{1}} \frac{\partial \Psi^{\ell_{1}}}{\partial \rho^{1}} \frac{\partial v^{\ell_{1}}}{\partial \Psi^{\ell_{1}}}\right)\right. \\
\left.-\cos \left(\alpha-\theta^{\ell_{2}}\right)\left(v^{\ell_{2}}\left(\Psi^{\ell_{2}}(\rho)\right)+\rho^{\ell_{2}} \frac{\partial \Psi^{\ell_{2}}}{\partial \rho^{\ell_{2}}} \frac{\partial v^{\ell_{2}}}{\partial \Psi^{\ell_{2}}}\right)\right)^{2}
\end{gathered}
$$

$+4 \rho^{\ell_{1}} \rho^{\ell_{2}} \cos \left(\alpha-\theta^{\ell_{1}}\right) \cos \left(\alpha-\theta^{\ell_{2}}\right)\left(\frac{\partial \Psi^{\ell_{1}}}{\partial \rho^{\ell_{2}}} \frac{\partial v^{\ell_{1}}}{\partial \Psi^{\ell_{1}}}\right)\left(\frac{\partial \Psi^{\ell_{2}}}{\partial \rho^{\ell_{1}}} \frac{\partial v^{\ell_{2}}}{\partial \Psi^{\ell_{2}}}\right)$

is positive for all $\alpha \in[0,2 \pi[$.

Lemma: The system (1) is hyperbolic for all $\rho^{\ell_{1}}, \rho^{\ell_{2}} \in$ $\left[0, \rho_{\max }\right]$ if and only if the vector directions of flow are collinear and have same directions.

An illustration of the area of the model hyperbolicity for the different level of congestion is displayed in Figure 3.
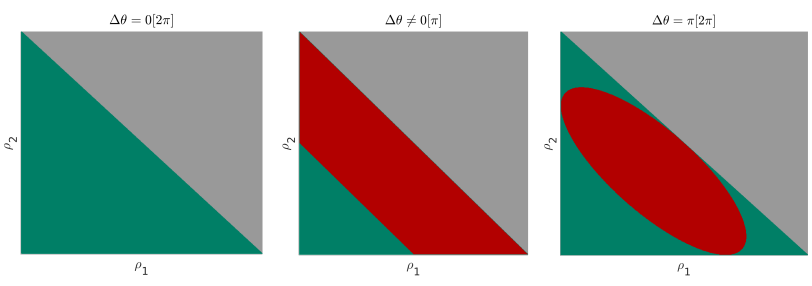

Fig. 3. Domain of density where the model is hyperbolic (green) or elliptic (red) for angles in the same direction, in different directions or in opposite direction

In this paper, the fluxes of the two layers have different directions. Thus, system (1) is not hyperbolic but hyperbolicelliptic depending on the density. The non-hyperbolicity of the model can be an issue for the stability of the model. In particular, it has been shown in [12] than a loss of hyperbolicity can lead to oscillation. In this paper, we do not have theoretical results on the stability of the model but we investigate if a higher order numerical scheme can help avoiding these kinds of oscillation in the numerical solution.

\section{B. Numerical method: high order schemes}

There is no analytical solution for Equation (1) so the solution has to be obtained numerically. Space is discretized with a rectangular and regular grid with a step $\Delta x$. The time is discretized uniformly with a step $\Delta t$. We denote by $C_{i, j}$ the cells of the grid and by $\rho_{i, j}$ the discrete density.

In order to avoid the numerical issue mentioned above, we consider a numerical method coupling the WENO scheme, the total variation diminishing (TVD) Runge-Kutta time discretization and the dimensional splitting. It is a high order method in space and time: fifth order in space, third order in time. At the cell interfaces, the numerical flux considered is Lax-Friedrichs.

We introduce first the notion of the different methods and then we present the final numerical method that combines these different parts.

The Weighted Essentially Non-Oscillatory (WENO) is a high order numerical method that is able to achieve both accuracy in smooth regions and to maintain sharp discontinuity and non-oscillatory transition of the solution. The flux at the interfaces can be chosen among all the usual one. In our case, we decide to use the Lax-Friedrichs Flux:

$$
F_{i+\frac{1}{2}, j}=\frac{1}{2}\left(\Phi\left(\rho_{i+1, j}^{-}\right)+\Phi\left(\rho_{i, j}^{+}\right)-v_{\max }\left(\rho_{i, j}^{+}-\rho_{i+1, j}^{-}\right)\right) .
$$

Contrary to first order method, with the WENO method, the value of the density at the interface $\rho_{i+1, j}^{-}$and $\rho_{i, j}^{+}$are not set equal to the density of the closest cell but estimated by a spatial polynomial interpolation of the five neighboring densities. We refer to [21] for a detailed description of the method.

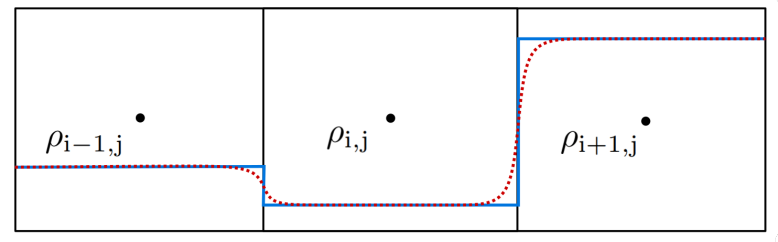

Fig. 4. Illustration of the WENO method: WENO interpolation (in red) of the discrete density (in blue)

An illustration of the method is shown in Figure 4. To avoid drawbacks in the interpolation close to strong discontinuities, we consider the adaptation for the interpolation as described in [21].

The dimensional splitting is a method that computes a numerical flux in several dimensions by splitting of the different dimension components. The flux is computed first in one dimension, then all the densities are updated and the flux is computed in the second dimension. More details on this method can be found in [22].

The scheme can be described as follows $\forall i, j$ :

$$
\begin{aligned}
& \rho_{i, j}^{i n t}=\rho_{i, j}^{\text {prev }}+\frac{\Delta t}{\Delta x}\left(F_{i-\frac{1}{2}, j}^{\text {prev }}-F_{i+\frac{1}{2}, j}^{\text {prev }}\right) \\
& \rho_{i, j}^{\text {next }}=\rho_{i, j}^{\text {int }}+\frac{\Delta t}{\Delta y}\left(F_{i, j-\frac{1}{2}}^{i n t}-F_{i, j+\frac{1}{2}}^{i n t}\right)
\end{aligned}
$$


where $F_{i+\frac{1}{2}, j}$ is the numerical flux at the interface between two cells $C_{i+1, j}$ and $C_{i, j}$. The numerical flux considered is the one of Lax-Friedrich as described in Equation (6). The subscript (prev, int, next) represent the different steps of the scheme and the density considered to evalued each flux. We denote by $\mathrm{L}$ the operator that represent the evolution of the dimensional splitting such that

$$
L: \rho_{i, j}^{\text {prev }} \rightarrow \rho_{i, j}^{\text {next }}
$$

as described in Equation (7). The Figure 5 gives a visual representation of the two steps of the dimensional splitting.
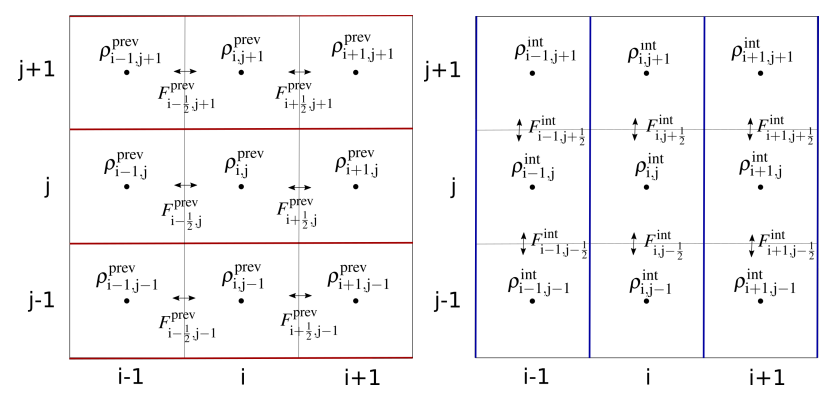

Fig. 5. Description dimensional splitting: on the left, the propagation is done for every line along the $\mathrm{x}$-dimension and on the right, the propagation if done for every column along the y-dimension.

For simplification of the scheme, the top subscript have not been represented in the Figure 5 .

The discretization in time of the scheme is done by the popular total variation diminishing (TVD) Runge-Kutta time discretization methods which are third order accurate. The idea is to divide the evolution of each time step in three and can be described as follow:

$$
\begin{aligned}
& \rho^{*}=\rho^{n}+\Delta t L\left(\rho^{n}\right) \\
& \rho^{* *}=\frac{3}{4} \rho^{n}+\frac{1}{4} \rho^{*}+\frac{1}{4} \Delta t L\left(\rho^{*}\right) \\
& \rho^{n+1}=\frac{1}{3} \rho^{n}+\frac{2}{3} \rho^{* *}+\frac{2}{3} \Delta t L\left(\rho^{* *}\right)
\end{aligned}
$$

where $L$ denote the numerical method developed in Equation (8) (dimensional splitting coupled with WENO method).

Finally, for each layer of density, each time step of the numerical scheme proposed is divided into six steps: three steps correspond to the TVD filter introduced in Equation (9) themselves divided into two steps by the dimensional splitting (propagation in $\mathrm{x}$ and $\mathrm{y}$ direction). For each of the substeps, the evolution of the densities is computed coupling Lax-Friedrich Numerical Flux and WENO interpolation. A visual representation is given in Figure 6.

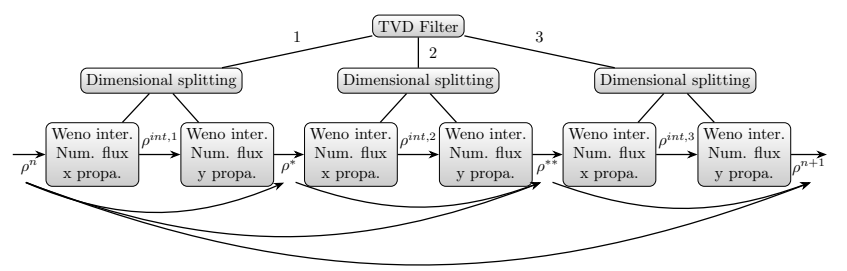

Fig. 6. Dependence scheme for the numerical method

\section{Simulation RESUltS FOR DIFFERENT STUDY CASES INCLUDING DIFFERENT SCENARIOS}

The advantage of a physical dynamic model compared with data based models is the ability to test scenarios that occurred only a few times in the past and take a decision according to the result.

\section{A. Study case 1: Delay in the traffic demand}

In this study case, we aim to test how a delay in the traffic demand can improve traffic status. We assume that there are two main streams in the network. One is directed from the South-East to the North-West and the other from the South-West to the North-East. If the main part of traffic demand starts at the same time, then it leads to congestion in the center. For this reason, we investigate a scenario in which we delay the demand of one stream and compare the result with the simulation without intervention. The time horizon of the simulation, $T_{\text {horizon }}$ is 30 minutes.

Lets denote by $L_{\mathrm{x}}$ and $L_{\mathrm{y}}$ the length of the network side. The initial condition is set by defining an initial density in the network and with no inflow:

$$
\left\{\begin{array}{l}
\rho_{0}^{1}(x, y)=\frac{e^{-\frac{C_{a}}{10}}}{4}+\frac{e^{-\frac{C_{b}}{10}}}{4}+\frac{e^{-\frac{C_{c}}{10}}}{4} \\
\rho_{0}^{2}(x, y)=\frac{e^{-\frac{C_{d}}{10}}}{4}
\end{array},\right.
$$

where

$$
\begin{gathered}
C_{a}=\sqrt{\left(x-0.2 L_{\mathrm{x}}\right)^{2}+\left(y-0.4 L_{\mathrm{y}}\right)^{2}} \\
C_{b}=\sqrt{\left(x-0.4 L_{\mathrm{x}}\right)^{2}+\left(y-0.2 L_{\mathrm{y}}\right)^{2}} \\
C_{c}=\sqrt{\left(x-0.25 L_{\mathrm{x}}\right)^{2}+\left(y-0.25 L_{\mathrm{y}}\right)^{2}} \\
C_{d}=\sqrt{\left(x-0.75 L_{\mathrm{x}}\right)^{2}+\left(y-0.25 L_{\mathrm{y}}\right)^{2}}
\end{gathered}
$$

We assume that the operator of the network give advice to the users of the layer 1 to depart their trip 6 min later to avoid congestion. The aim of this study case is to compare a scenario, where $90 \%$ of the layer 1 users follow the advice given by the operator, with a normal scenario, where no advice is given. The results of the simulation and comparison with the control experiment are presented in Figure 7.

The strategy applied allows desynchronizing the high density of vehicles crossing from different origin and then avoid the creation of congestion in the middle of the network. There is less congestion in the scenarios with late departure. We can notice that the vehicles waiting for departure are displayed in the density of Figure 7 but do not impact traffic condition or travel time statistics. This is to say that the time due to the delay is not lost for drivers.

For the scenarios with and without control, the total number of drivers that carry out a trip is equal to 24 470. A trip corresponds to the trajectories of drivers from their initial position to the outside of the network. The average number of vehicles within the network over the time horizon is equal to 7374 for the scenario without intervention from the operator and 6340 for the scenario where the operator advice users 


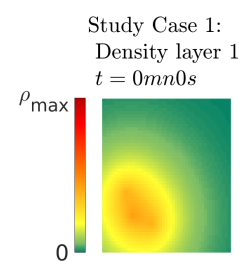

Nominal case

Density layer 2

$t=0 m n 0$

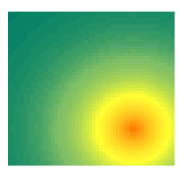

$t=1 \mathrm{mn} 0 \mathrm{~s}$

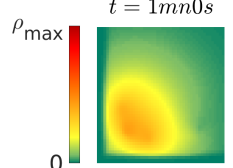

$t=1 m n 0 s$

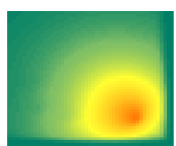

$t=5 \mathrm{mn} 0 \mathrm{~s}$

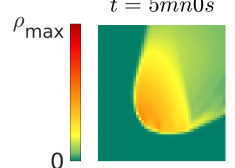

$t=5 \mathrm{mn} 0 \mathrm{~s}$

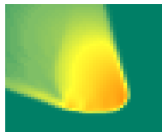

$t=7 m n 30$

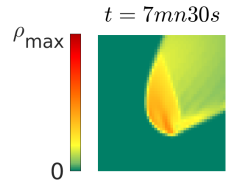

$t=15 \mathrm{mn} 0 \mathrm{~s}$

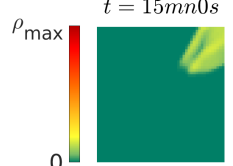

0

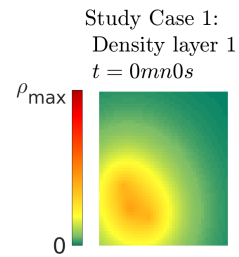

Density layer 2

$t=0 \mathrm{mn} 0 \mathrm{~s}$
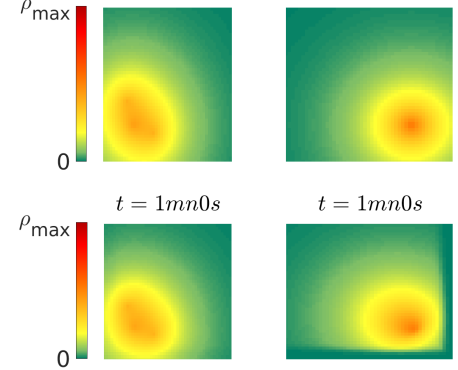

$t=1 m n 0 s$

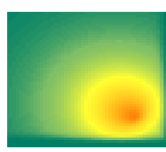

$t=5 m n 0 s$

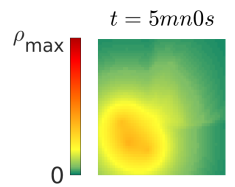

$t=7 m n 30 s$

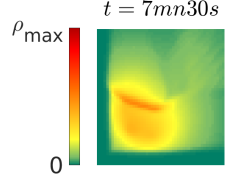

$t=15 \mathrm{mn} 0 \mathrm{~s}$
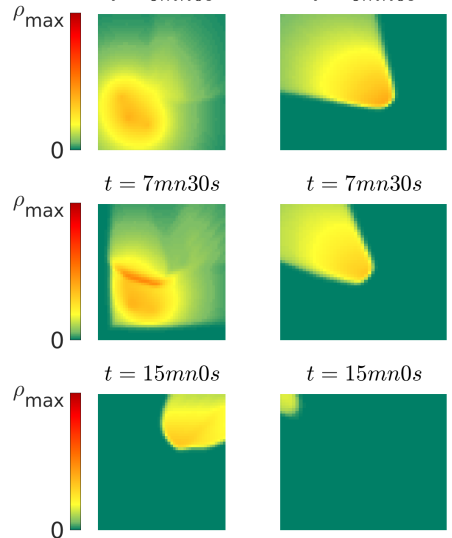

$t=7 m n 30 s$

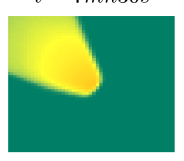

$t=15 \mathrm{mn} 0 \mathrm{~s}$
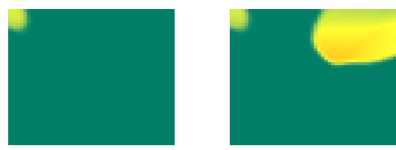

Fig. 7. Simulation results of the study case 1, on the top for the nominal case and on the bottom for the scenario with control by driver advice (delay departure). The left column corresponds to the layer of density 1 , the middle column of the layer 2 and the right column to the total density. A video of the complete simulation is available Online at https://youtu.be/ Jvt0eGI4D 7c. to leave later. From these numbers, the average travel time of drivers can be deduced as follow:

$$
\text { Average trip time }=\frac{\text { Average number of user }}{\text { Total number of user }} \times T_{\text {horizon }}
$$

The results of the simulation are summed up in Table I. In this table, TTT represents the Total Traveling Time of drivers. The average travel time of users without advice of

\begin{tabular}{|l|c|c|}
\hline & Nominal case: No delay & Delay in departure \\
\hline Number trip & 24470 vehicles & 24470 vehicles \\
Average trip time & $9 \mathrm{mn} 02 \mathrm{~s}$ & $7 \mathrm{mn} 46 \mathrm{~s}$ \\
TTT & 3687 hours & 3170 hours \\
\hline
\end{tabular}

TABLE I

COMPARISON OF THE RESULTS OF SIMULATION FOR THE STUDY-CASE: DELAY IN DEMAND

the operator (late departure) is equal to $9 \mathrm{mn} 02 \mathrm{~s}$. With the advice of the operator, the average travel time decreases to $7 \mathrm{mn} 46$. In total for all the users, this corresponds to 517 hours of congestion saved namely a reduction of $14 \%$ of the total travel time.

\section{B. Study case 2: Congestion due to a protest march}

In this study case, we aim to predict how a protest march can impact traffic condition. A zone corresponding to this protest march is defined in the North-Center of the network and we assume that no vehicle can enter this area.

Lets denote by $L_{\mathrm{x}}$ and $L_{\mathrm{y}}$ the length of the network side. The initial condition is set by defining an initial density in the network:

$$
\left\{\begin{aligned}
\rho_{0}^{1}(x, y) & = \begin{cases}\frac{6}{16} \rho_{\max } & \text { if }(x, y) \in D_{a} \\
0 & \text { otherwise }\end{cases} \\
\rho_{0}^{2}(x, y) & = \begin{cases}\frac{3}{16} \rho_{\max } & \text { if }(x, y) \in D_{b} \\
0 & \text { otherwise }\end{cases}
\end{aligned}\right.
$$

where

$$
\begin{aligned}
& D_{a}=\left\{(x, y) \in \Omega, \frac{2}{16} L_{\mathrm{x}} \leq x \leq \frac{7}{16} L_{\mathrm{x}} \& \frac{2}{16} L_{\mathrm{y}} \leq y \leq \frac{7}{16} L_{\mathrm{y}}\right\} \\
& D_{a}=\left\{(x, y) \in \Omega, \frac{9}{16} L_{\mathrm{x}} \leq x \leq \frac{14}{16} L_{\mathrm{x}} \& \frac{2}{16} L_{\mathrm{y}} \leq y \leq \frac{7}{16} L_{\mathrm{y}}\right\}
\end{aligned}
$$

and with a constant inflow equal to $\frac{4}{25} v_{\max } \rho_{\max }$ on the network boundary $\partial \Omega$. The time horizon of the simulation is 30 minutes.

The aim of this study case is to analyze the impact of the protest march by considering different scenarios: one with no protest march, the second one with a small protest march and the last one with a large protest march. The results of the simulation and comparison between the case with and without protest march are shown in Figure 8. We can notice that congestion is created in the 3 scenarios. However, the level of congestion reached is significantly different. If the scenario without protest march is considered as the reference, 
we can remark that the average density of the scenario with a small protest march is $11 \%$ higher and then the average density of the scenarios with a large protest march is $21 \%$ higher.

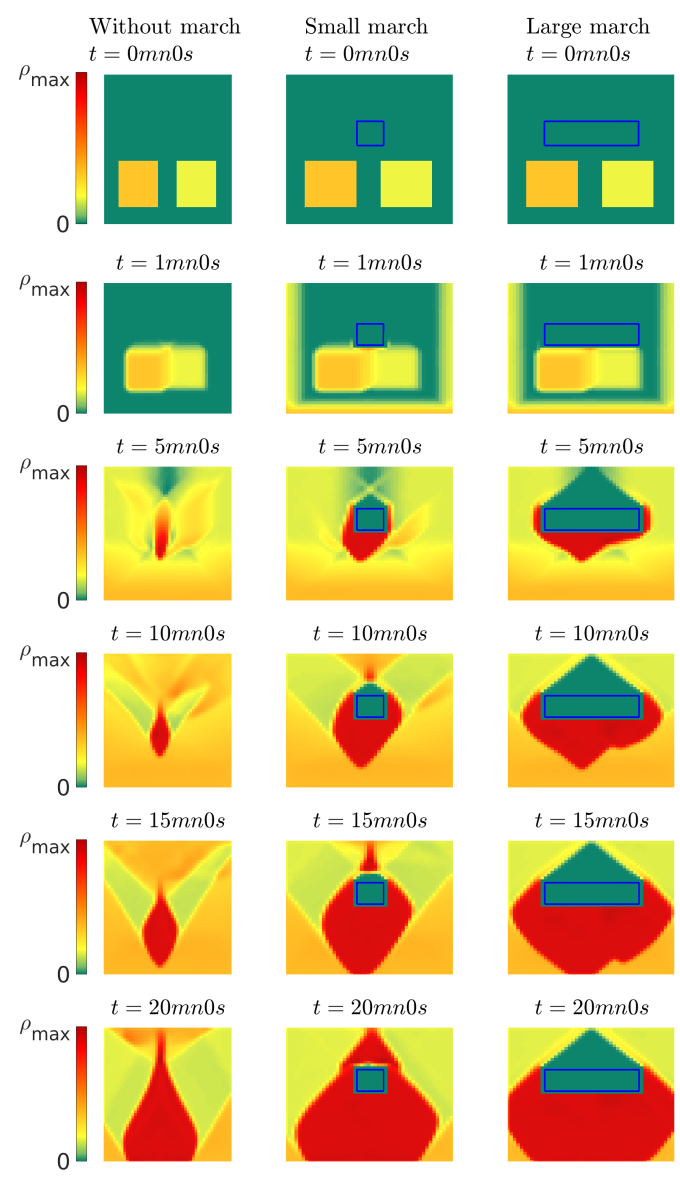

Fig. 8. Results of a simulation comparing the total density of 3 scenarios for the study case 2 of a protest march: on the left the scenario with no protest march, on the middle with a small protest march and on the right with a large protest march. A video of the complete simulation is available Online at https://youtu.be/nkieROrzBiI.

\section{CONCLUSION}

We have presented a two dimensional and multi-layer traffic model that is able to describe both to describe traffic in large scales and with multiple directions of flows. Higher order numerical method WENO has been considered for the simulation. Different study-cases including each different scenarios have been analyzed, showing for what kind of decision this model can potentially be useful in the future for traffic operator.

\section{ACKNOWLEDGMENT}

This project has received funding from the European Research Council (ERC) under the European Unions Horizon 2020 research and innovation programme (grant agreement 694209).

\section{REFERENCES}

[1] S. Mollier, M. L. Delle Monache, and C. Canudas-de-Wit, "A simple example of a two-dimensional model for traffic: Discussion about assumptions and numerical methods," Transportation Research Record, vol. 2672 , no. 20 , pp. 249-261, 2018.

[2] — , "2D-LWR in large-scale network with space dependent fundamental diagram," in 201821 st International Conference on Intelligent Transportation Systems (ITSC), 2018, pp. 1640-1645.

[3] T. L. Friesz, K. Han, P. A. Neto, A. Meimand, and T. Yao, "Dynamic user equilibrium based on a hydrodynamic model," Transportation Research Part B: Methodological, vol. 47, pp. 102-126, 2013.

[4] S. Samaranayake, W. Krichene, J. Reilly, M. L. D. Monache, P. Goatin, and A. Bayen, "Discrete-time system optimal dynamic traffic assignment (SO-DTA) with partial control for physical queuing networks," Transportation Science, vol. 52, pp. 982-1001, 2018.

[5] Y. Lv, Y. Duan, W. Kang, Z. Li, F.-Y. Wang et al., "Traffic flow prediction with big data: A deep learning approach." IEEE Trans. Intelligent Transportation Systems, vol. 16, no. 2, pp. 865-873, 2015.

[6] M. J. Lighthill and G. B. Whitham, "On kinematic waves. ii. a theory of traffic flow on long crowded roads," in Proceedings of the Royal Society of London A: Mathematical, Physical and Engineering Sciences, vol. 229, no. 1178. The Royal Society, 1955, pp. 317-345.

[7] P. I. Richards, "Shock waves on the highway," Operations research, vol. 4, no. 1, pp. 42-51, 1956.

[8] B. D. Greenshields, J. Thompson, H. Dickinson, and R. Swinton, "The photographic method of studying traffic behavior," in Highway Research Board Proceedings, vol. 13, 1934.

[9] M. Garavello, K. Han, and B. Piccoli, Models for vehicular traffic on networks. American Institute of Mathematical Sciences (AIMS), Springfield, MO, 2016, vol. 9.

[10] N. Geroliminis and C. F. Daganzo, "Existence of urban-scale macroscopic fundamental diagrams: Some experimental findings," Transportation Research Part B: Methodological, vol. 42, no. 9, pp. 759770, 2008.

[11] L. Leclercq, C. Parzani, V. L. Knoop, J. Amourette, and S. P. Hoogendoorn, "Macroscopic traffic dynamics with heterogeneous route patterns," Transportation Research Procedia, vol. 7, pp. 631-650, 2015

[12] P. Goatin and M. Mimault, "A mixed system modeling two-directional pedestrian flows," Mathematical biosciences and engineering, vol. 12, no. 2, pp. 375-392, 2015.

[13] Y. Jiang, S. Wong, H. Ho, P. Zhang, R. Liu, and A. Sumalee, "A dynamic traffic assignment model for a continuum transportation system," Transportation Research Part B: Methodological, vol. 45, no. 2, pp. 343-363, 2011.

[14] J. Du, S. Wong, C.-W. Shu, T. Xiong, M. Zhang, and K. Choi, "Revisiting jiang's dynamic continuum model for urban cities," Transportation Research Part B: Methodological, vol. 56, pp. 96-119, 2013.

[15] L. Romero Perez and F. G. Benitez, "Outline of diffusion advection in traffic flow modeling," in Transportation Research Board 87th Annual Meeting, no. 08-1503, 2008.

[16] M. M. Khoshyaran and J.-P. Lebacque, "Homogeneous bidimensional traffic flow model," IFAC-PapersOnLine, vol. 51, no. 9, pp. 61-66, 2018

[17] M. Herty, A. Fazekas, and G. Visconti, "A two-dimensional data-driven model for traffic flow on highways," Networks and Heterogeneous Media, vol. 13, pp. 217-240, 2018.

[18] S. Mollier, M. L. Delle Monache, C. Canudas-de-Wit, and B. Seibold, "Two-dimensional macroscopic model for large scale traffic networks," Transportation Research Part B: Methodological, vol. 122, pp. 309 326, 2019.

[19] Z. Lin, S. Wong, P. Zhang, Y. Jiang, K. Choi, and Y. Du, "A predictive continuum dynamic user-optimal model for a polycentric urban city," Transportmetrica B: Transport Dynamics, vol. 5, no. 3, pp. 228-247, 2017.

[20] E. Tory, H. Schwandt, R. Ruiz-Baier, and S. Berres, "An adaptive finite-volume method for a model of two-phase pedestrian flow," Networks and Heterogeneous Media, vol. 6, no. EPFL-ARTICLE170240, pp. 401-423, 2011.

[21] C.-W. Shu, "High order weighted essentially nonoscillatory schemes for convection dominated problems," SIAM review, vol. 51, no. 1, pp. 82-126, 2009.

[22] K.-A. Lie, "A dimensional splitting method for quasilinear hyperbolic equations with variable coefficients," BIT Numerical Mathematics, vol. 39 , no. 4, pp. 683-700, 1999. 